أثر التدريس بطريقة النمذجة بِ تعديل تصورات طالبات الصف الحادي عشر البديلة لمفاهيم حفظ الطاقة وكمية التحرك

عبدالله خميس امبوسعيدي* ورحمة محمد الصابري

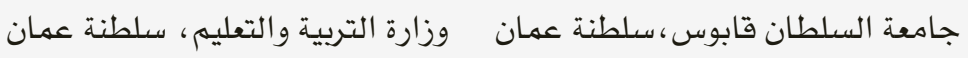

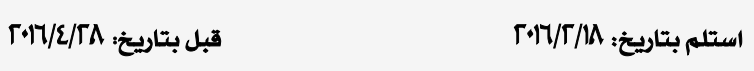

ملخص: هدفت الدراسة إلى تقصي أثر التدريس بطريقة النمذجة ِِّ اكتساب مفاهيم حفظ الطاقة وكمية التحرك وتعديل تصورات

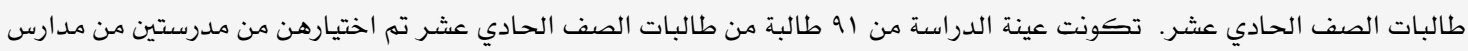

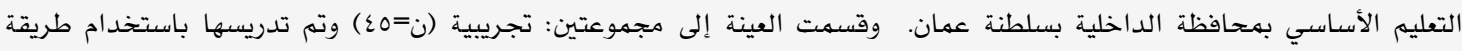
النمذجة، وضابطة (ن=حـ) تم تدريسها بالطريقة السائدة، وقد استفرقت المعالجة التجريبية حوالي ستة أسابيع خلال الفصل الدراسي

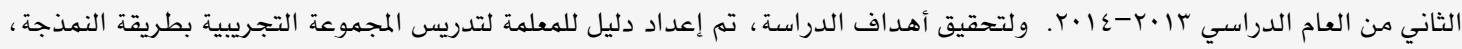

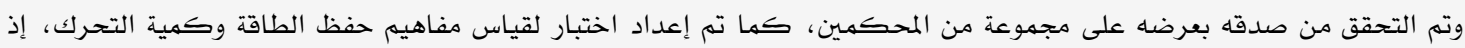

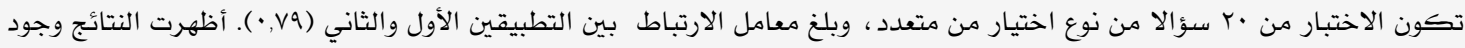

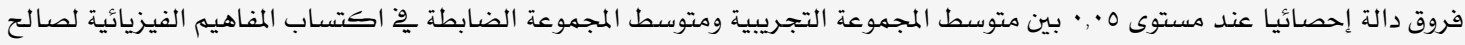

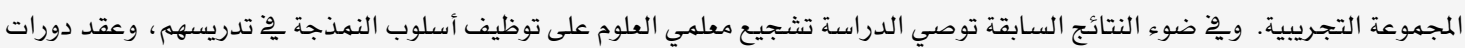
تدريبية وورش عمل بوّكيفية تطبيق النماذج للمعلمين والمشرفين والقائمين على إعداد المناهج. كلمات مفتاحية: التدريس بالنهذجة ، التصورات البديلة ، مفاهيم حفظ الطاقة وكمية التحرك، طالبات الصف الحادي عشر.

\title{
The Impact of Teaching Physics via Modeling on Modifying Alternative Conception of Energy and Momentum Conservation among 11th Grade Female Students
}

\author{
Abdulah K. Ambusaidi* \\ $\&$ \\ Rahma M. Al Sabri \\ Sultan Qaboos University, Sultanate of Oman Ministry of Education, Sultanate of Oman
}

\begin{abstract}
This study investigated the impact of teaching physics via modeling on the acquisition of energy and momentum conservation concepts. The sample consisted of 91 female students selected from 11th grade female students in two schools in Al-Dakhiliyah Governorate in Oman. The experimental group $(\mathrm{n}=45)$ was taught via the modeling method, and the control group $(\mathrm{n}=46)$ was taught using a traditional method. The study lasted six weeks during the second semester of the academic year 2013/2014. A teacher guide for teaching by models was designed and validated by a group of experts. To measure the acquisition of physics concepts, energy and momentum, an achievement test was used. The test consisted of 20 multiple-choice questions. Its reliability was measured by test-retest method $(\mathrm{r}=0.79)$. The results revealed a statistically significant difference $(\mathrm{p}<.05)$ between the means of the experimental and control groups in favor of the experimental group. The study recommends that science teachers ought to use models and modeling in their teaching. Workshops to train supervisors, in-service teachers and pre-service teachers in the construction and development of scientific models need to be conducted.
\end{abstract}

Keywords: Teaching via Modeling, conservation of energy and momentum, Alternative Conception, 11th grade female students.

*ambusaid@squ.edu. om 
تفسير الظواهر التي تتطلب ريط بين عدد من المفاهيم Halloun, 2001; ) المكونة للنظريات والقوانين الفيزيائية (Mercan, 2012; Perkins et al, 2004

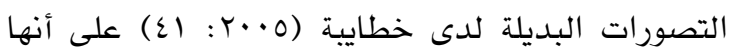
"تفسير غير مقبول - وليس بالضرورة خطأ -للظواهر الطبيعية، يقدمه المتعلم نتيجة المرور بخبرات حياتية، أو

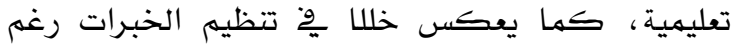
كونها نتيجة لعمليات نشطة، ومقصودة كتلك التي

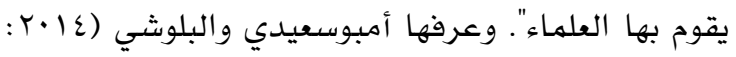

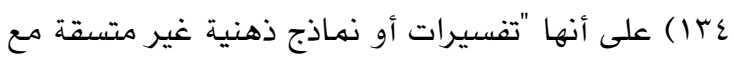

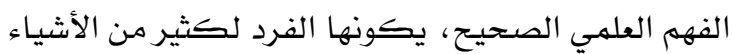
التي يريد أن يتعلهها بحيث تكون بعيدة عن سياقها الصحيح ضمن فئة أخرى مختلفة عنها". ورغم أهمية مفاهيم حفظ الطاقة وكمية التحرك باعتبارها إطارا أساسيا ومهما يوْ تدريس الميكانيكا

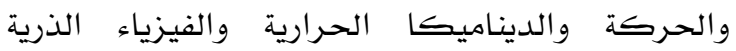
والنووية، كما أنها ساهمت بِّ اكتشاف بعض التقانات والأجهزة ِِّ إنتاج الطاقة، وِّْ تصميم معدات وأجهزة

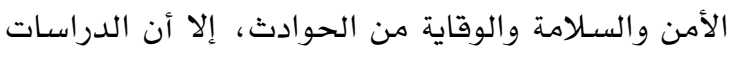

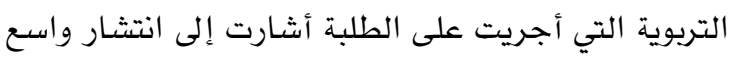

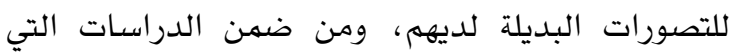

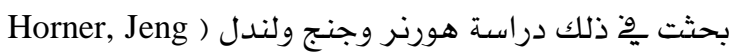
(\& Lindell, 2007 الني تشير إلى صعوبة توظيف مفاهيم حفظ الطاقة وكمية التحرك يجّ المسائل المفاهيمية التي لتي لا تعتمد على الحسابات الرياضية، أما دراسة الحة لعائ

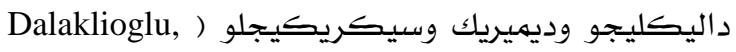
التي أجريت على ع^ال (Demirici \& Şekercioglu, 2015 طالبا وطالبة من طلبة الصف الحادي عشر پِّ تركيا، فقد أشارت نتائجها إلى معاناة الطلبة ِِّ الريط بين مفاهيم حفظ الطاقة وكمية التحرك، وبالتالي صعوبة توظيفها بِّ المواقف المختلفة، مها ولد تصورات بديلة

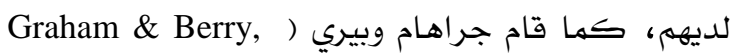
1996) بدراسة عينة مكونة من جعه طالبا وطالبة من طلبة المرحلة الثانوية ، هدفت إلى الكشف عن فهم الطلبة لمفهوم كمية التحرك الذي يتمثل وِّ العلاقة بين الكتلة

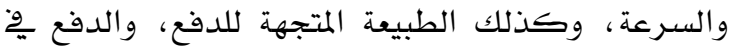

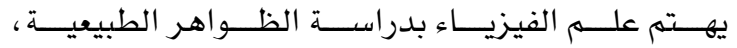

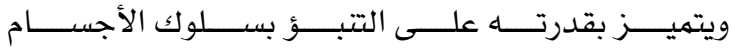

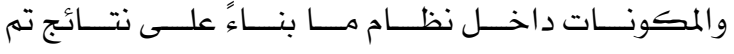

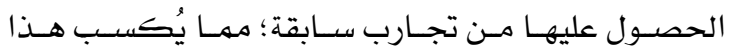

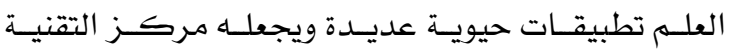

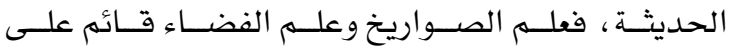
قـوانين جـاليليو ونيـوتن بشــكل رئيس، ووســائل النقـل

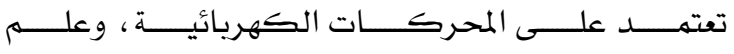

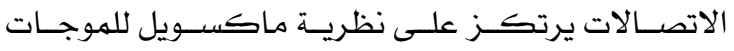

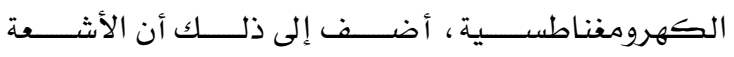

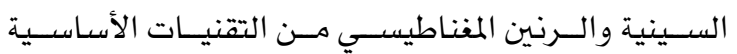

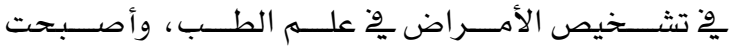

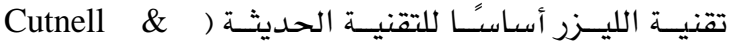
.(Johnson, 2010 ورغم تلك الأهمية للمفاهيم الفيزيائية، إلا أن المتعلمين يجدون صعوبة پِّ اكتسابها، ويشير كل من كوبرن

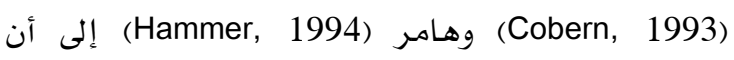
صعوبة فهم مادة الفيزياء لدى الطلبة تتمثل هوّ: التمييز بين المفاهيم المختلفة وربط بعضها ببعض، وتوظيف تلك فلكة المفاهيم والمبادئ الأساسية بِّ المواقف الحقيقية لتفسير الظواهر الطبيعية. ويرجع ذلك إلى عدة أسباب: منها ما يتصل بطبيعة المفاهيم الفيزيائية التي تتسم بالصعوبة التية والتجريد (Mäntylä, 2011)، وأخرى تتعلق بالبيئة

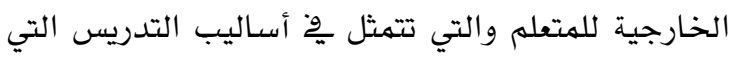

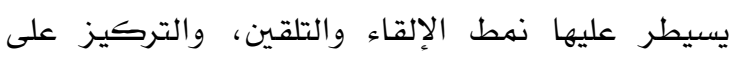
المعالجات الرياضية دون الاهتمام بالمعالجات المفاهيمية، وهناك أسباب تتعلق بطبيعة المتعلم من حيث تدني

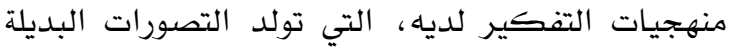
(Alternative Conception)

.$(Y \cdots O$

أشار ميركان (Mercan, 2012) إلى أن امتلاك المتعلم للتصورات البديلة يؤدي إلى صعوبة تطبيقه للهفاهيم الفيزيائية؛ إذ يستخدم ِّو حالات كثيرة معادلات رياضية لتوضيح بعض الظواهر الطبيعية وتفسيرها. ويعود ذلك

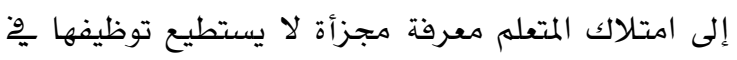


ومفهوم كمية التحرك، وانتقال الطاقة ِوْ أثناء التصادمات.

ومن أجل التفلب على تلك الصعوبات وتعديل التصورات البديلة لدى الطلبة ، يسعى المعلمون جاهدين إلى استخدام طرق تدريسية تساعد على تتظيم المعرفة العلمية على شكل نهاذج وأنهاط شاملة لدى الطلبة (زيتون، • • ب؛ واتخاذ (Halloun, 1996; Seballa \& Redish, 2007 أساليب توضح العلاقة بين الجانب التربوي والجانب الإبستمولوجي، وتبرز دور فهم طبيعة العله يخ توجيه عمليتي التعليم والتعله (Sin, 2014)، إذ أن النظرة الإبستمولوجية للمفهوم الفيزيائي قائمة على افتراضين رئيسين، هما: أن المتعلم يبني تعلمها بنفسـه من خلال تفاعله هع بيئة التعلم المحيطة باه، وأن المعرفة عملية تكيفية تحدث من خلال تتظيم الفرد للخبرات التي يتفاعل معها (Mathews, 1997). وتعد النماذج والنمدجة من الإستراتيجيات الفعالة يخٌ اكتسـاب المفاهيم العلمية، ، وتعديل التصورات البديلة لدى الطلبة.

Gilbert ) والنموذج هو تمثيل لظاهرة أو لجسم أو لفكرة

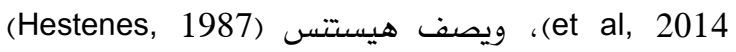
النموذج العلمي بأنه تمثيل للنظام الذي يتكون من مادة أو مجموعة من المواد تجمع بينها صفات وخصائص تشكل نهطا مشتركا. وأضاف هلون (Halloun, 2011) أن الأنماط تظهر ِِ النماذج المفاهيمية كما تظهر تماها پِّ النماذج المادية، فالنهوذج المادي يعبر عن مجموعة أجسام مادية تتفاعل فيها بينها وتعكس نمطا معينا يوخ تركيبها وسلوكها مثل: الذرة والخلية، والجهاز العصبي، والنظام الشهسي، أما النموذج المفهومي فيعبر عن الكيانات الذهنية المجردة المرتبطة مع بعضها البعض، التي تعكس نهطا معينا ِِّْ العالم المعربِّ مثل: النظرية العلمية، والقاعدة العلمية، والمبدأ العلهي. Halloun, ) ويشتمل النموذج المفهومي كما يراه هلون

$$
4 \text { :2011: } 4
$$

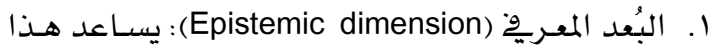
البُعد على تحديد المعرفة العلميـة المتمثلة يوْ المفـاهيم والقوانين والمبادئ الخاصة بالنموذج وبنيته. ويتكون

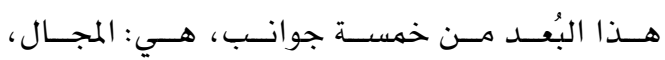

بعد واحد ، ومبدأ حفظ كمية التحرك ِّْ بعدين، وبناء على نتائج الدراسة صنف الطلبة وفق أربع فئات، وهي: عدم القدرة على التهييز بين المفاهيم، وإتقان المفاهيم الأسـاسية وتوظيفها يُّ بعض المواقف بدون إدراك العلاقة بين كمية التحرك والدفع، وإدراك أن كمية التحرك كمية متجهة ، ومعرفة العلاقة بينها وبين الدفع وفق نظرية كمية التحرك والدفع، التي تؤدي إلى فهم مبدأ حفظ كمية التحرك بِّ بعد، وآخر فئة لديها فهم شامل لمفهوم كمية التحرك وعلاقته بالدفع ببعديها. كما أجرى سيركينجلو (Cirkinoglu, 2004) مرجع غير مدرج ِ2 الثبت دراسة شبه تجريبية على طلبة المرحلة المتوسطة والثانوية، واستخدم مقياسـا يحتوي على أسئلة مفتوحة تكشف عن مدى فهم الطلبة لمفاهيم كمية التحرك والدفع، وكذلك أجرى مقابلة لبعض الطلبة، وأشارت النتائج إلى وجود تصورات بديلة لدى طلبة المجموعة التجريبية والضابطة.

كها أشـارت عدد من الدراسات إلى وجود تصورات بديلة لدى الطلبة يوٌ مبدأ حفظ الطاقة، وبالتالي صعوبة فهم انتقال الطاقة وانسيابها بِّ2 الأنظمة ( ) clldowie, 1995) Chabalengula et al, 2012; McDermott, 1997 غير مدرج ِِّ الثبت). وأشـار كل من سنج وروسينجرت إلى الصعوبة التي يواجهها (Singh \& Rosengrant, 2003) الطلبة بِّ توظيف مفاهيم حفظ الطاقة وكمية التحرك عِّ تفسير بعض الظواهر الطبيعية نتيجة وجود تصورات بديلة لدى الطلبة.

George, أما دراسة جورج وبرودستوك وفازكويز عباد Broadstock \& Vazquez Abad, 2000 فقد استهدفت تقصي أثر إستراتيجيتين من إستراتيجيات التدريس هما : المختبرات الافتراضية المبنية على المستوى الدقائقي، وتحليل نتائج التجارب بِ الفيديوهات الرقمية. وأشـارت نتائج الدراسـة إلى أثر الإستراتيجيتين ِِّْ اكتساب المفاهيم الخاصة بالتصادمات ومبادئ الحفظ، واستخدام الطلبة للتكنولوجيا. كها أشارت إلى وجود صعوبات بح فهم حفظ كمية التحرك وحفظ الطاقة ، وخاصة مفهوم

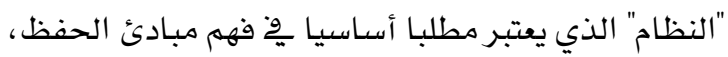


الأدوات والمواد والوسائل التعليمية (Halloun, 2004)، ويهثل الشكل (1) مراحل دورة النمذجة.

\section{5 النموذجي}

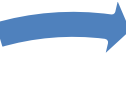

\section{1.الاستكشاف}

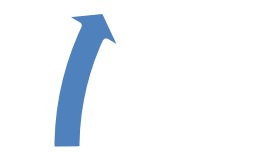

$$
\text { 4 النموذبيق }
$$

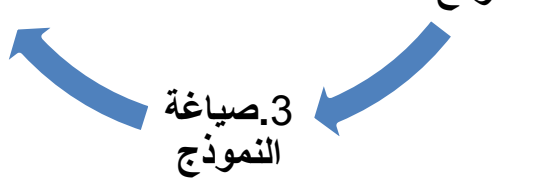

شكل ا : مراحل دورة النتمذجة

يتضح من الشكل ا أن دورة النمذجة دورة مطورة من

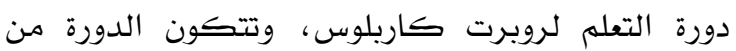
خمس مراحل، تبدأ بهرحلة "الاستكشاف" التي لترني يكتشف فيها الطالب إمكانات النموذج الذي بني سابقا وحدوده، كما أنه يدرك وِّهذ هذه المرحلة الحاجة إلى بناء نموذج جديد، بعد ذلك يوجه المعلم الطلبة إلى مرحلة

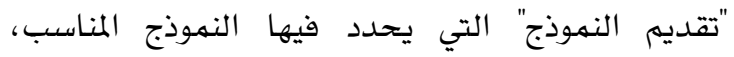

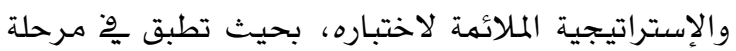

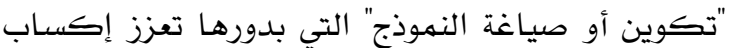
المفهوم للطالب؛ لكي يتم تطبيقه وِّ مرحلة "تطبيق

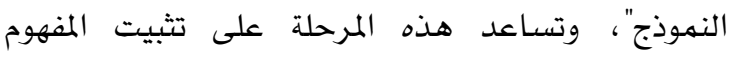

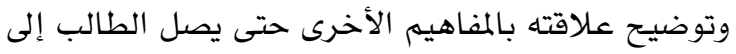

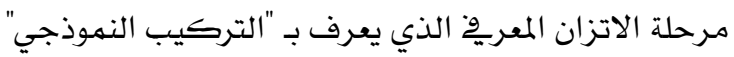
الذي يوضح الاتساق بين الأنساق الفكرية التي يمتلكها

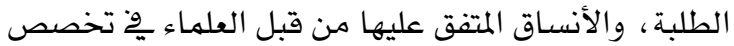

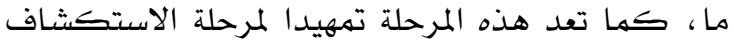

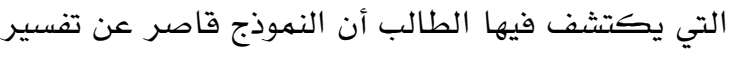

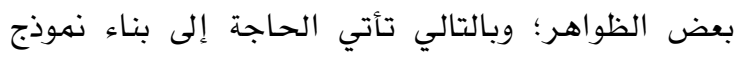

جديد (Halloun, 1996).
والوظيفة، والبنية ، والتركيب الداخلي، والتركيب

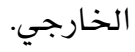

r. البُعـد الإدراكـي (Cognitive dimension): يسـاعد هذا البُعد على تحديد مهارات التفكير اللازمة لبناء

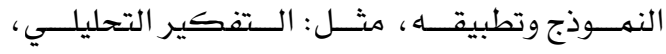
والتفكير الترابطي، والتفكير الناقد، والتفكير

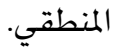

r. البُعد السلوكي (Behavioral dimension): يركِّز هــذا البُعـد علـى تحديـــ مهـارات الأداء، والمهـارات

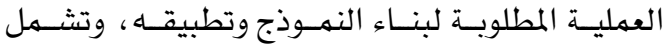

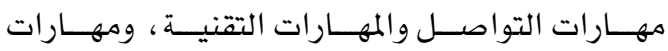
التشغيل، والمهارات الفنية ، ومهارات التفاعل البيئي. ع. البُعـد الميتـا إدراكي (Metacognitive dimension): يهدف هذا البُعد إلى ضبط تفاعل الطلبة مـع الأبعاد

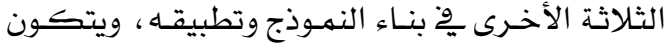

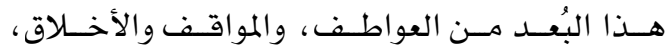
والآداب، والقيه، ووجهات النظر ، والاعتقادات.

إن استخدام النماذج ِِّ تدريس العلوم يشجع الطلبة على

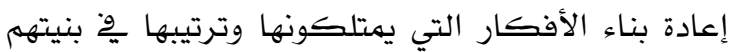
المعرفية، كما يساهم على توليد نهاذج ذهنية ناجحة، وهذا ما أكدته عدد من المنظمات والمؤسسات الدولية التي دعت إلى تبني التدريس المبني على النماذج، وتوظيف

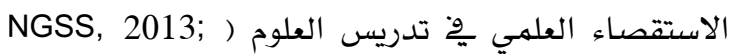
(NSTA, 1995 كما يعد استخدام النماذج عامل ربط بين التجرية

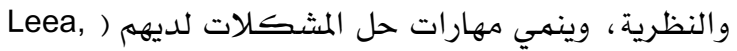
Jonassenb, \& Teo, 2011

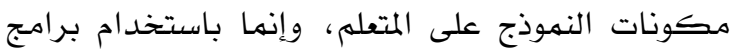
نمذجة مختلفة توضح التظيم المنطقي للمعارف، وتعد

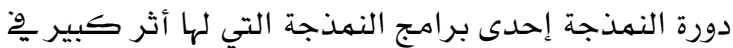

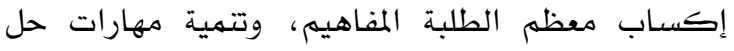
المشكلات لديهم، حيث يستطيع المعلم البدء من أي بعد المدابه

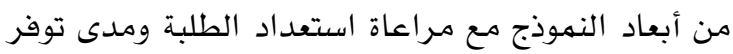


r. ما أثر التدريس بالنمذجة ِِّ تعديل التصورات البديلة لدى طالبات الصف الحادي عشر عن بن مفاهيم حفظ الطاقة وكمية التحرك؟ لكال

فرضية الدراسة

تمت صياغة فرضية واحدة مرتبطة بالسؤال الأول وهي أنه لا يوجد فرق ذو دلالة إحصائية عند مستوى 0 •. - وِ2

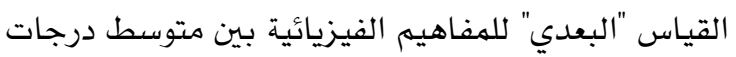
طالبات الصف الحادي عشر من المجموعة التجريبية وطالبات المجموعة الضابطة.

\section{أهمية الدراسة ومبرداتها}

تبرز أهمية الدراسـة ِِّْ أنها:

1. تستخدم طريقة جديدة من طرق تتظيم المعارف وِ2

تدريس العلوم بشكل عام والفيزياء بشكل

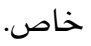

r. تقتح المجال أمام دراسات أخرى تتناول التدريس

بالنمذجة وعلاقتها بهتفيرات تعليمية -تعلمية

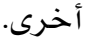

r. تقدم لمعلهي مادة الفيزياء للصف الحادي عشر

دليلا يتضمن تطبيقات بِّ استخدام طريقة تتظيم

$$
\text { المعارف وفق النماذج. }
$$

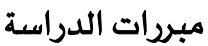

1. ضرورة إكساب المفاهيم الفيزيائية بصورة

صحيحة، وتعديل التصورات البديلة لدى الطلبة،

لذا تتوافق هذه الدراسة مع التوجهات العالمية بِّ

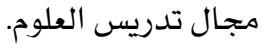

r. الحاجة إلى الاهتمام بالتصورات البديلة التي

يهكن أن يكونها الطلبة عند تدريس المفاهيم الماهيم

العلمية.

r. الاستجابة لكثير من توصيات البحوث

والدراسات على المستوى العالمي والعربي والمحلي

التي تدعو إلى ضرورة إكساب الطلبة مفاهيم

حفظ الطاقة وكمية التحرك وتعديل التصورات
مشكلة الدراسة وأسئلتها

يمثل تعلم مادة الفيزياء تحديا لدى كثير من طلبة المرحلة الثانوية؛ لاحتوائها على عدد من المفاهيم والمبادئ والقوانين التي يعتقدون أنها لا تمت لهم بصلة سواء بو

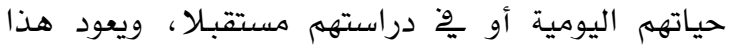
الاعتقاد إلى وجود ضعف پِّْ اكتسابهم لتلك المفاهيم

وصعوبة ربط بعضها ببعض (Halloun, 2001: Hammer, 1994; Koponen, 2007; McDermott, 1993; Mercan, 2012; Perkins et al, 2004; Reif \&

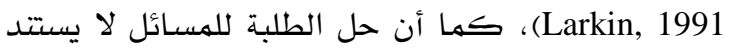
على فهم جيد واستيعاب شامل للمفاهيم والمبادئ والقوانين الفيزيائية؛ مما يسبب لديهم صعوبة پِّ تقسير الظواهر الطبيعية نتيجة امتلاكهم لتصورات بديلة سواء "ِّْ استيعابهم للمفاهيم أو قدرتهم على الربط بين المفاهيم المكونة للقوانين والمبادئ المستخدمة پِّ تقسير تلك الظواهر ) Cutnell \& Johnson, 2010; Hestenes, 1997 .(Wells, Hestenes \& Swackhamer, 1995 ووِّ ظل ذلك أصبح من الضروري دراسة طبيعة تلك المفاهيم وطرق الوصول إليها، وطرق تعليمها وتعلمها.

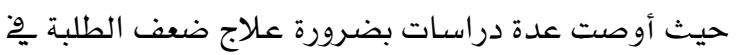

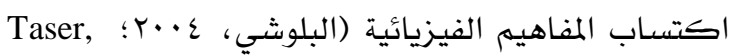
2010؛ Philippi, 2010 ؛ Clement, 1982 ، وتعديل Mclldowie, 1995; ) التصورات البديلة لديهم ،Chabalengula et al, 2012; McDermott, 1997 وأكدت بعض المؤسسات والمنظمات الدولية على أهمية تبني التدريس القائم على النماذج بِّْ اكتساب المفاهيم العلمية (NGSS, 2013; NSTA, 1995)، كما أشارت بعض الأدبيات إلى أهمية التظيم المعربِّ القائم على المانى

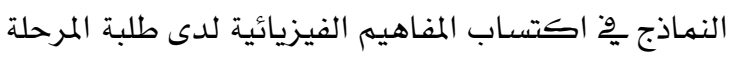
Hestenes, 1995; Jackson, Dukerich, ) الثانوية Hestenes,2008; Leea, Jonassenb \& Teo, 2011; (Ornek, 2008 وعليه سعت الدراسة الحالية إلى الإجابة عن السؤالين

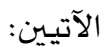

ا. ما أثر التدريس بالنمذجة ِِخ اكتساب مفاهيم حفظ الطاقة وكمية التحرك لدى طالبات الصف

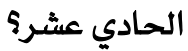


اقتصرت النماذج على البعد المعرِِّ فقط والذي يتم فيه تتظيم المفاهيم والقوانين والمبادئ

والنظريات حسب مكونات النموذج الخمسة ،

وهي: المجال، والوظيفة، والبنية، والتركيب

$$
\text { الد اخلي والتركيب الخارجي. }
$$

\section{التعريفات الاصطلاحية والإجرائية}

لهذه الدراسـة مجموعة من المصطلحات من المهم تعريفها إجرائيا ، وهي:

النموذج The Model يعرفه هلون (Halloun, 2011: 3 (i) على أنه "الكيانات الذهنية والمجردة المرتبطة مع بعضها البعض التي تعكس نمطا معينا ِِّْ العالم المعربِّ، مثل: النظرية العلمية ، والقاعدة العلمية، والمبدأ العلمي. ويشهل النموذج أربعة أبعاد، هي: المفهومي، والإدراكي، والسلوكي، والميتا إدراكي. التدريس بالنمذجة (Teaching by Modeling): تشير بكلي (Buckley, 2012) مرجع غير مدرج ِ2ْ الثبت إلى أن التدريس بالنمذجة هو ذلك التدريس الذي يساعد المتعلم على بناء نهاذج ذهنية وتطويرها ، ويعرف الباحثان التدريس بالنمذجة بِّو الدراسة الحالية على أنها عملية

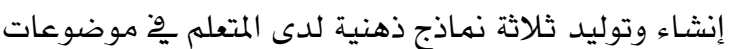
حفظ الطاقة وكمية التحرك؛ إذ يبنى كل نموذج وفق مكوناته الخمسة: المجال، والوظيفة، والبنية، والتركيب الداخلي، والتركيب الخارجي. وذلك باستخدام دورة النمذجة لكل نهوذج، وفق مراحلها الخمسة: الاستكشاف، وتقديم النموذج، وصياغة النموذج، وتطبيق النموذج، والتركيب النموذجي.

المفاهيم العلمية (Scientific Concepts): يعرف زيتون

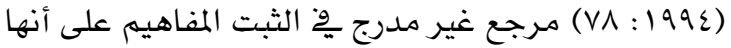
"ما يتكون لدى الفرد من معنى وفهم يرتبطان بكلمة مصطلح أو عبارة أو عملية معينة". ويعرف الباحثان

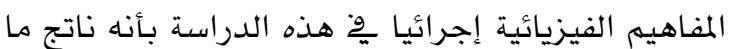
اكتسبته الطالبة من بنى مفاهيمية سواء كانت مفاهيم أو قوانين ومبادئ وقواعد ترتبط بتلك المفاهيم، وتطبيقاتها الحياتية المتضهنة ِِ وحدة "حفظ الطاقة
البديلة لديهم ( Mclldowie, 1995; Chabalengula .(et al, 2012; McDermott, 1997

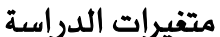

تتحدد متغيرات الدراسة الحالية فيما يلي: المتغير المستقل، وهو طريقة التدريس وله مستويان هما :

$$
\text { التدريس بالنمـجة. }
$$

التدريس بالطريقة السائدة.

$$
\text { المتغير التابع، ويتمثل ِِّ؛: }
$$

اكتساب الطلبة للهفاهيم الفيزيائية ِِّْ مفاهيم

حفظ الطاقة وكمية التحرك.

تعديل التصورات البديلة ِِّ مفاهيم حفظ الطاقة

$$
\text { وكمية التحرك. }
$$

حدود الدراسة

تهثلت حدود هذه الدراسة التي تحد من تعميمها بِّ أنها : اقتصرت على موضوعات الفصل الخامس (حفظ الطاقة) والفصل السادس (حفظ كمية التحرك) من وحدة حفظ الطاقة وكمية التحرك، التي يستخدمها كتاب الفيزياء للصف الحادي عشر بسلطنة عمان. اقتصرت على متغير اكتساب الطلبة لمفاهيم حفظ الطاقة وكمية التحرك المتضهنة يُّوحدة وحدة الطاقة وكمية التحرك وعلى تعديل التصورات البديلة ِِّ هذه المفاهيم.

تم تطبيقها ِِّْ الفصل الدراسي الثاني من العام

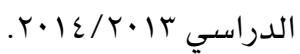

اقتصرت على عينة من طالبات الصف الحادي عشر پِّ مدرستين من مدارس التعليم ما بعد الأسـاسي (الصفين || وY||) بسلطنة عمان. تتحدد نتائجها بمدى صدق اختبار اكتساب المفاهيم الفيزيائية المعد لغرض الدراسـة. 
طالبة درسن وحدة حفظ الطاقة وكهية التحرك بالطريقة السائدة. وقد جاء اختيار هاتين المدرستين لمناسبة ظروف المدرستين لتطبيق الدراسة، ووجود معلمات ذوات خبرة ومستوى تدريسي متقارب، وذلك بناء على ترشيح مشرفيهم التربويين. وقد اختيرت معلمتان من مدرستين مختلفتين وذلك حتى لا ينتقل أثر التدريس بطريقة النمذجة من المجموعة التجريبية إلى المجهوعة

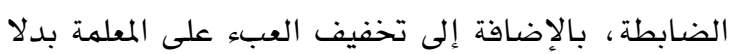
من التدريس بطريقتين مختلفتين ولمجموعتين مختلفتين ووِّْ الفترة الزمنية نفسها.

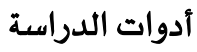

دليل المعلم: بعد الاطلاع على بعض الدراسات والأدبيات المختلفة سواء تلك التي احتوت على طريقة إعداد الأنشطة القائمة على النمذجة مثل كتاب نظرية النمذجة ِون تدريس العلوم لهلون (Halloun, 2004)، أو تلك التي تختص بتظيم موضوعات الفيزياء وفق النماذج مثل كتاب "المقدمة بو الفيزياء" لكيوتينل وجونسون (Cutnell \& Johnson, 2010)، أو تلك التي تحتوي على \&لى أنشطة وتجارب بديلة تتعلق بهوضوعات حفظ الطاقة وكمية التحرك وتطبيقاتها المختلفة وِّ الحياة اليومية والموجودة ِِّ شبكة المعلومات العالمية "الإنترنت"، تم تحديد البنى المفاهيمية المتضهنة ِوْ تلك المراجع، التي تتناسب مع وحدة حفظ الطاقة وكمية التحرك وِّ كتاب الفيزياء للصف الحادي عشر ومستوى طالبات الصف، ثم أعيد تتظيم تلك البنى المفاهيمية وفق مكونات النموذج الآتية: المجال، والبنية، والوظيفة، والتركيب الداخلي، والتركيب الخارجي. وتم تضمـين النموذج وفق الخطوات الخمس للدورة نمدجة: الاستكشاف، بداية تكوين النموذج، صياغة النموذج،

$$
\text { تطبيق النموذج، التركيب النهوذجي. }
$$

والجدير بالذكر أنه يهكن تتظيم الموضوعات الخاصة بالحركة والديناميكا -وهي من أفرع الفيزياء

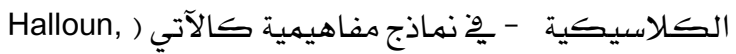

$$
\begin{aligned}
& \text { 1. نهوذج الجسم المتسـارع بانتظام. } \\
& \text { r. نموذج الحركة التوافقية البسيطة. }
\end{aligned}
$$

وكمية التحرك" من كتاب الفيزياء ِِ الصف الحادي عشر، وسيتم قياسها إجرائيا من خلال الدرجة التي ستحصل عليها الطالبة يخ اختبار اكتساب المفاهيم الفيزيائية الذي سيتم إعداده من خلال ترجمة مقياس Energy and المستح المفاهيهي للطاقة وكمية التحرك لكميك Momentum Conceptual Survey (EMCS)

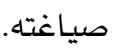

التصورات البديلة Alternative Misconceptions):

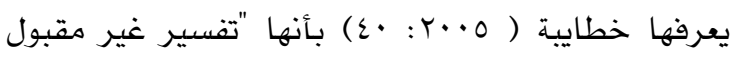
وليس بالضرورة خطأ للظواهر الطبيعية يقدمـه المتعلم نتيجة مروره بخبرات حياتية أو تعليمية ، كما يعكس خلا بِّ تتظيم الخبرات رغم كونها نتيجة لعمليات نشطة وبنائية ومقصودة كتلك التي يقوم بها العلماء" وتم تحليل التصورات البديلة ِّْ هذه الدراسة وفق نسب البدائل الصحيحة لجميع أسئلة الطالبات وِّ المجموعتين، ومقارنة تلك النسب وفق القياس القبلي والبعدي، وذلك وفق

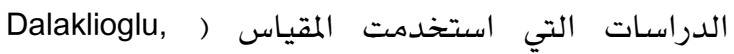
Demirci, Sekercioglue, 2015; Singh \& Rosengrant, 2003)، كما تم قياس معدل الاكتساب بين المجموعتين التجريبية والضابطة وقياس التصورات البديلة باستخدام

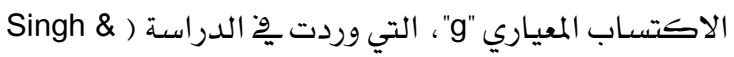
(Rosengrant, 2003

$$
g=\frac{s_{f}-s_{i}}{100-s_{i}} \quad \text { الآتية }
$$

حيث i $\quad$ ت تمثل نسبة البدائل الصحيحة قبل إجراء المعالجة ، و $S_{f}$ تهثل نسبة البدائل الصدحيحة بعد المعالجة.

\section{عينة الدراسة}

تكونت عينة الدراسة من (91) طالبة من طالبات الصف الحادي عشر تم اختيارهن من مدرستين من مدارس

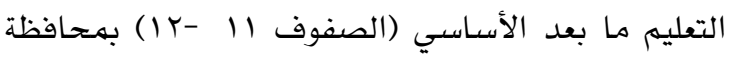

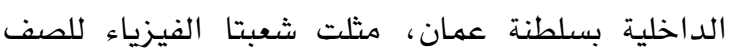
الحادي عشر ِِّ إحدى المدرستين بالمجموعة التجريبية، حيث بلغ عدد طالبات هذه المجموعة (0) طالبة درسن وحدة حفظ الطاقة وكمية التحرك باستخدام طريقة التدريس بالنمذجة، وكذلك شعبتي الفيزياء للصف الحادي عشر ِِّ المدرسة الأخرى تمثلان المجموعة

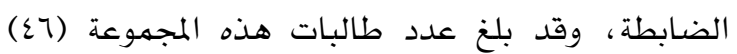


كما تم تضمـين نهاذج حاسوبية توضح أشكال الطاقة وتحولاتها، ومبدأ حفظ الطاقة، ومبدأ حفظ كمية التحرك.

\section{اختبار اكتساب مفاهيم حفظ الطاقة وكمية التحرك:}

استقاد الباحثان من مقياس المستح المفاهيهي للطاقة وكمية التحرك Energy and Momentum Conceptual (EMSV) Survey وروسينجرانت (Singh \& Rosengrant, 2002) پِّ إعداد اختبار مفاهيم حفظ الطاقة وكمية التحرك الذي يتضمن على خمسة وعشرين سؤالا من نوع اختيار من متعدد، يلي كل سؤال خمسـة بدائل، بنيت تلك الأسئلة وفق تصنيف بلوم لمستويات المعرفة. وقد قلصت عدد البدائل إلى أربعة بدائل لكل سؤال؛ وذلك بها يتوافق مع مواصفات أسئلة الاختيار من متعدد المتبعة من قبل وزارة التربية والتعليم، ويهثل كل بديل خاطئ تصورا بديلا لدى الطالبة، إذ حددت التصورات البديلة ِِّْ مفاهيم الطاقة وكمية التحرك من خلال بحث أجراه كل من

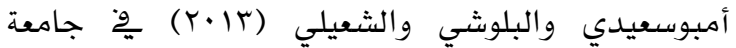
السلطان قابوس، وكذلك البحث الذي أخذ منه المقياس (Singh \& Rosengrant, 2002)، وبعض البحوث التي لتوني اهتمت بدراسة مفاهيم حفظ الطاقة وكمية التحرك Mclldowie, 1995; Chabalengula et al, 2012; )

(McDermott, 1997

وللتأكد من صدق اختبار المفاهيم الفيزيائية تم عرضه پِّ صورته الأولية على عدد من المحكمـين من ذوي الخبرة

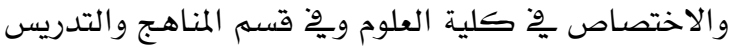
بكلية التربية بجامعة السلطان قابوس، ومشرِوْ مادة

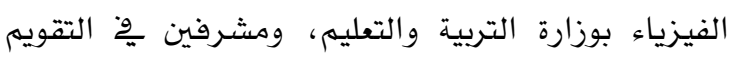
التربوي، وقد طلب منهم إبداء مـلاحظاتهم حول صياغة الأسئلة، وسـلامتها علميا ولغويا، وتحديد مدى صعوبتها ومناسبتها لطالبات الحادي عشر. وللتحقق من ثبات الاختبار، تم تطبيقه على عينة مكونة من (ro) طالبة من طالبات الصف الحادي عشر من خارج عينة الدراسـة الأصلية بِ و الفصل الدراسي الثاني من العام

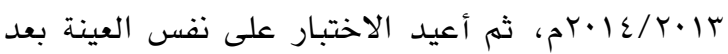

$$
\begin{aligned}
& \text { r. نهوذج الجسم تحت تأثير الدفع. } \\
& \text { ع. نموذج الحركة الدائرية المنتظمة. } \\
& \text { o. نهوذج دوران المجسمات. }
\end{aligned}
$$

حيث توضتح أسماء النماذج السابقة البنية الأسـاسية للنموذج وهجال تطبيقه، ويهكن استخدام النهوذج الواحد يوّ توضيح أكثر من ظاهرة أو مفهوم أو مبدأ أو ونجا نظرية، ويعتمد ذلك على بنية النموذج وتركيبه أو كليهما معا ، وعلى ذلك يعطى كل نهوذج عنوانا وفق الموضوع الذي يتتاوله. وبالنسبة لنماذج وحدة حفظ الطاقة وكمية التحرك فقد تم تتظيمها وفق ثلاثة نهاذج كالآتي:

النموذج الأول: وهو نموذج الجسمى المتسـارع بانتظام، الذي يتناول العلاقة بين الشغل (المبذول بواسطة قوة ثابتة) والطاقة، علما بأن الطالبات قد درسن ِِّ الصف العاشر الشغل والطاقة ولكنهن لهم يدرسن العلاقة بينهما، ويضهم هذا النموذج سبعة موضوعات، وهي: الثغل المبذول بواسطة قوة ثابتة، ونظرية الثغل - الطاقة وطاقة الحركة، وطاقة الوضع التثاقلية (الشغل المبذول بواسطة الجاذبية)، والقوى التي تسبب حفظ النظام والقوى التي لا تسبب حفظ النظام، ومبدأ حفظ الطاقة الميكانيكية، والقدرة، وصور أخرى للطاقة ومبدأ

حفظ الطاقة.

النموذج الثاني: وهو نهوذج الحركة التوافقية البسيطة للجسه، الذي يتتاول الشغل المبذول بواسطة قوة متغيرة، وهنا تأتي الحاجة إلى حساب الشغل بيانيا ، حيث يتكون هذا النموذج من موضوع واحد فقط وهو الشغل المبذول بواسطة قوة متغيرة وكيفية حسابه. النموذج الثالث: وهو نموذج الجسم تحت تأثير الدفع، الذي يتاول العلاقة بين الدفع وكمية التحرك، ويتكون من أربعة موضوعات، هي: نظرية الدفع -كمية التحرك، ومبدأ حفظ كمية التحرك الخطية، والتصادمات ِِّْ بعد واحد ، والتصادمات ِِّْ بعدين. 
نفسها ولكن بالطريقة السائدة التي اعتادت المعلمة على تدريس طالباتها بها.

المتوسطات الحسابية والانحرافات المعيارية وقيمة (ت)

لمتوسط درجات المجموعتين التجريبية والضابطة وِية

اختبار المفاهيم الفيزيائية القبلي الدرجة الكلية ِِوِ الاختبار= • · ب درجة.

يتضح أنه لا توجد فروق ذات دلالة إحصائية بين

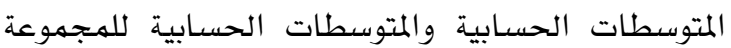
الضابطة ِِّ التحصيل القبلي پِّ اختبار حفظ الطاقة وكمية التحرك، وبالتالي يهكن القول: إن طالبات المجموعتين التجريبية والضابطة متكافئتان قبل البدء تطبيق المعالجة التجريبية.

\section{نتائج الدراسة ومناقشتها}

للإجابة عن السؤال الأول الذي ينص على "ما أثر التدريس بطريقة النمذجة ِ2ْ اكتساب مفاهيم حفظ الطاقة وكمية التحرك لدى طالبات الصف الحادي عشرى" تم تطبيق اختبار اكتساب مفاهيم حفظ الطاقة وكمية التحرك بعديا بعد الانتهاء مباشرة من تدريس احسبا المبـ وحدة حفظ الطاقة وكمية التحرك على مجموعتي الدراسـة، وحسـاب المتوسطات الحسابية والانحرافات المعيارية لمتوسطي درجات طالبات المجموعتين التجريبية والضابطة، واستخدم اختبار (ت) للعينتين المستقلتين لحساب دلالة الفروق بين المتوسطات الحسابية للهجموعتين التجريبية والضابطة وِّ اختبار المفاهيم

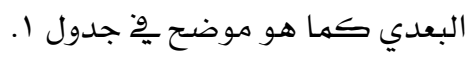
يتبين من جدول ا أنه توجد فروق ذات دلالة احصائية عند مستوى 0., · بين متوسطات درجات طالبات مجموعتي الدراسة ِوْ اختبار المفاهيم الفيزيائية ككل لصالح المجموعة التجريبية، حيث بلغ المتوسط الحسابي الكلي لنتائج طالبات المجموعة التجريبية ِوخ اختبار المفاهيم

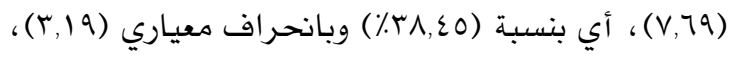
وِخ حين أن المتوسط الحسابي لطالبات المجموعة الضـابطة

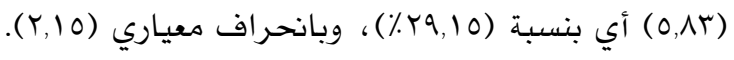
وهذا يعني أنه تم رفض فرضية الدراسة الصفرية ، والتي
أسبوعين من التطبيق الأول. وبحساب معامل ارتباط بيرسون وجد أنه يساوي (V9, · ) وهي قيمة جيدة وصالحة ولحة لأغراض الدراسـة.

\section{طريقة تدريس المجموعتين}

تم تدريس المجموعة التجريبية بطريقة النمذجة، حيث اتبعت المعلمة الخطوات المحدد لها يخّ دليل المعله المعد من قبل الباحثين والمتضهنة بِّ كيفية تطبيق طريقة التدريس بالنمذجة. إذ شرحت المعلمة مفاهيم الطاقة وكمية التحرك وفق مكونات النماذج الثلاثة المعدة ، وغيرت من تتظيم الدروس المتعلقة بالمفاهيم والقوانين والمبادئ المضهنة يْ كتاب الفيزياء للصف الحادي عشر. ومن ثم قدمت تلك النماذج وفق دورات نمدجة منتالية، حيث تم شرح كل نموذج وفق دورة نمذجة متكاملة بخطواتها الخمسـة ، التي تؤدي إلى بناء دورة نهذجة أخرى. أها طالبات المجموعة الضابطة فتم تدريسهن باستخدام التظيم المعربِّ المتعارف عليه ِِّ الكتاب، وذلك من قبل معلمة ذات خبرة ومستوى تدريسي متقارب مع معلمة

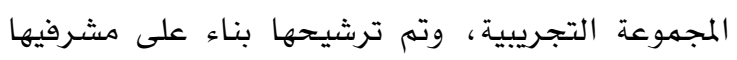
التربويين. وقد اختيرت معلمتان من مدرستين مختلفتين وذلك حتى لا ينتقل أثر التدريس بطريقة النمذجة من المجموعة التجريبية إلى المجموعة الضابطة، بالإضافة تخفيف على المعلمة بدلا من التدريس بطريقتين مختلفتين ولمجموعتين مختلفتين وِِّ الفترة الزمنية نقسها.

\section{الطريقة والإجراءات}

استخدمت هذه الدراسة المنهج شبه التجريبي، وذلك لأن عينة الدراسـة اختيرت قصديا لصفوف كاملة دون إجراء أي تعديل لأفراد العينة، كذلك وزعت الصفوف الأربعة بطريقة قصدية إلى مجموعتين تجريبية وضابطة وذلك حسب ظروف المدرستين اللتين تم اختيارهما. وطبق اختبار اكتساب المفاهيم الفيزيائية قبليا وبعديا على احئيا المجموعتين التجريبية والضابطة. وقد مرت المجموعة التجريبية بهعالجة عن طريق تدريسها وحدة حفظ الطاقة وكمية التحرك وفق التدريس بالنمدجة ولمدة (V) أسابيع وبهعدل ع حصص وِ كل أسبوع، وِ حين أن طالبات المجموعة الضابطة درسن الوحدة نفسها يُّ الفترة الزمنية 
المتوسطات الحسابية والانحرافات المعيارية وقيمة (ت) لمتوسط درجات طالبات المجموعتين التجريبية والضابطة وحجم

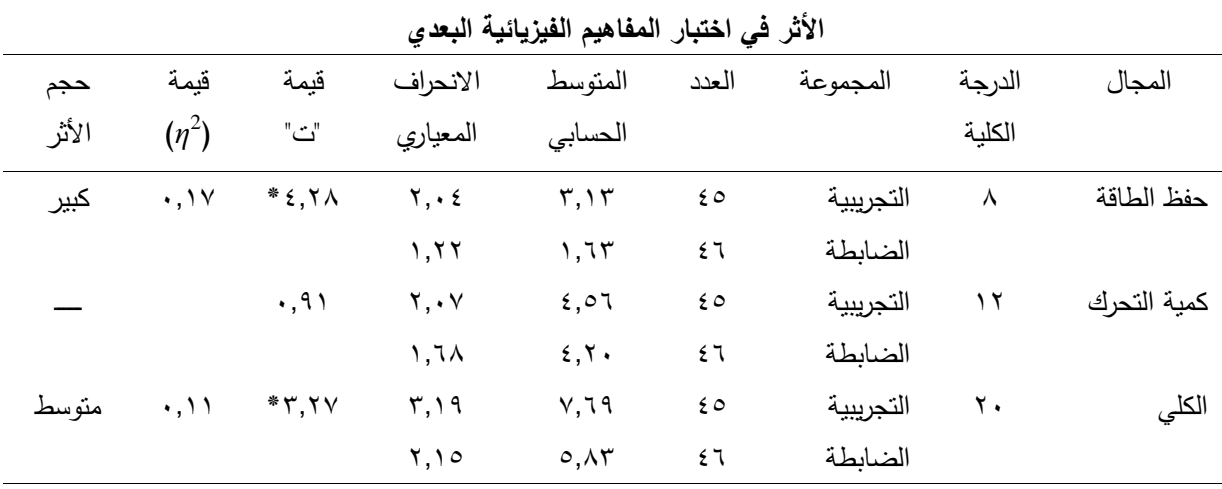

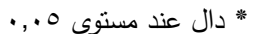

اكتساب طالبات الصف الحادي عشر للمفاهيم الفيزيائية، حيث أن كل مكون من مكونات النموذج الخمسة لله أهداف ومخرجات تعله محددة تسعى المعلمة إلى تحقيقها مع الطالبات وفق دورة النمدجة، أضف إلى ذلك تركيز الأنشطة على التعله المتمركز حول المتعلم، واتضح ذلك من خلال محاولة الطالبات بناء الفرضيات يخ بداية كل دورة، وهذا ما سـاعد على بناء النموذج بشكل متسلسل ومنطقي. ويتضهن التدريس بالنمذجة أنشطة تقصي تعاونية حسبها Wells, ) أشار كل من ويل وهيستس وسواكهمر Hestenes \& Swackhamer, 1995 الأنشطة على الحوار والمناقشة والعهل الجماعي، وساعد ذلك الطالبات على بناء الخرائط المفاهيمية التي توضح بنية النموذج وتركيبه المتمثلة وِ قوانين نيوتن وقوانين الحركة التوافقية البسيطة بشكل أساسي، وأدت الخرائط دورا ِِّ تقييم الطالبات بشكل مستهر، وكشف أي تصور بديل قد يتكون لديهن، فالنماذج أدوات تقييم أيضا كما أشار جلاين (Glynn, 1997) مرجع غير مدرج وِّا الثبت.

وتعد مرحلة التركيب النموذجي - وهي من مراحل دورة

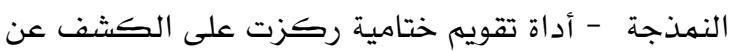
مدى فهم الطالبات لمكونات النموذج الخمسة، خلال مراحل دورة النمذجة. كما أن للخرائط المفاهيمية التي بنتها الطالبة تحت إشراف المعلمة المطبقة خلال دورة النمذجة ، وكذلك نشاط الشبكة والكلمات المتقاطعة
تص على أنه "لا يوجد فرق ذو دلالة إحصائية عند مستوى 0 •, · مِ القياس "البعدي" للمفاهيم الفيزيائية بين متوسط درجات طالبات الصف الحادي عشر هن المجموعة التجريبية وطالبات المجموعة الضابطة" وقبول الفرضية البديلة.أما فيما يتعلق بهجالي اختبار المفاهيم الفيزيائية وهما: حفظ الطاقة وكمية التحرك، فيتضح من جدول ا وجود فروق دالة احصائيا ِِّ مجال حفظ الطاقة لصالح المجموعة التجريبية، حيث بلغ المتوسط

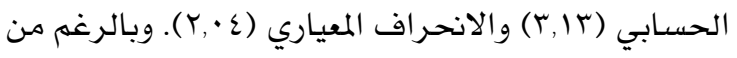
عدم وجود فروق دالة احصائيا ِِّ مجال كمية التحرك إلا أن متوسط درجات المجهوعة التجريبية أعلى هن متوسط درجات المجموعة الضابطة. كما تبين من جدول أن قيمـة مريع إيتا (2) للاختبار ككل بلفت

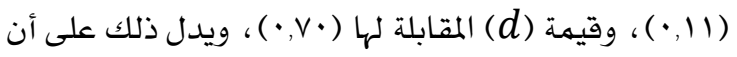
أثر طريقة التدريس بالنمذجة بِ اكتساب المفاهيم الفيزيائية لدى طالبات الصف الحادي عشر كان

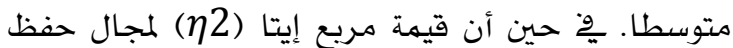
الطاقة بلفت (IV, •))، وقيهة (d) المقابلة لها (•q, ())، ويدل ذلك على أن أثر طريقة التدريس بالنمدجة ِِّ اكتساب مفاهيم حفظ الطاقة لدى العينة ذاتها كان

$$
\text { كبيرا. }
$$

ويعزى وجود فروق دالة احصائيا عند مستوى الدلالة بين متوسطي درجات طالبات المجمهوعتين التجريبية والضابطة وِ اختبار المفاهيم الفيزيائية إلى فاعلية تتظيهم موضوعات حفظ الطاقة وكمية التحرك وفق ثلاثة نماذج 


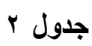

المفاهيم الرئيسية في وحدة حفظ الطاقة وكمية التحرك والأسئلة

المتعلقة بها

\begin{tabular}{|c|c|}
\hline الأسئلة & المفاهيم الرئيسة \\
\hline IV، ‘ ، I I & الثغل المبذول بواسطة الجاذبية \\
\hline $1961 Y ، 11 ،$ ك & مبدأ حفظ الطاقة الميكانيكية \\
\hline $1 \cdot r$ & مفهوم النظام \\
\hline \multirow[t]{2}{*}{11,114960} & حفظ كمية التحرك في التصادمات \\
\hline & المرنة وغير المرنة وعديمة المرونة \\
\hline V & مفهوم كمية التحرك \\
\hline 10 & العلاقة بين كمية التحرك والدفع \\
\hline$r \cdot .17$ & تطبيقات نظرية الدفع-كمية التحرك \\
\hline $1 \leqslant$ & مفاهيم الطاقة ومفاهيم كمية التحرك \\
\hline
\end{tabular}

يتضح من خلال جدول r أن الاكتسـاب المعياري المتوسط لطالبات المجهوعة التجريبية هو یז,· ، وتراوحت معدلات الاكتساب المعياري بين -0·, •و^乞,·• بينما الاكتساب المعياري لطالبات المجهوعة الضابطة هو ع •.• ، وتراوحت

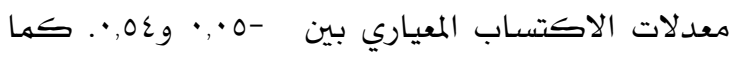
يتضح من خلال جدول ب أن استجابات الطلبة بو المجموعة التجريبية وِّ ثمانية أسئلة من بين ·ب سؤالا حصلت على أكثر من •ع٪٪، بينما عدد الأسئلة التي تفوق تلك النسبة يخٌ المجموعة التجريبية هي خهسة أسئلة من بين ·r سؤالا. وحاز السؤال الأول والأخير على أعلى

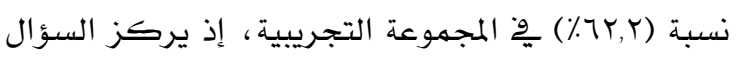
الأول على فهم الطالبات لمفهوم الشغل المبذول بواسطة الجاذبية الأرضية، ويركز السؤال ·r على توظيف الطالبات لنظرية الدفع -كمية التحرك، ومثل السؤال 19 أقل نسبة (10,7 \%) وْ استجابات الطالبات، ويعد أحد الأسئلة التي تكشف عن مدى فهم الطالبات لمبدأ حفظ الطاقة الميكانيكية. أها المجهوعة الضابطة، فهثل السؤال 10 أعلى نسبة

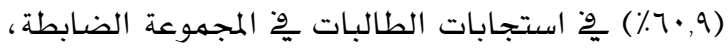
والذي يوضح العلاقة بين كمية التحرك والدفع، ويعد هذا السؤال من ضمن أسئلة مجال حفظ كمية التحرك

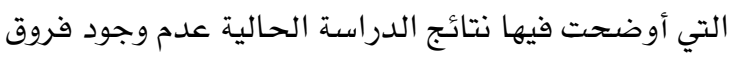
دالة إحصائيا بين المجموعتين كما بِّ جدول ب. ويعد أحد أسئلة مبدأ حفظ الطاقة الميكانيكية (السؤال 19) من أقل استجابات الطالبات وِ المجموعة الضـابطة ومثل نسبة الطالبات اللاتي أجبن عن هذا السؤال (7,0٪).
الواردة ِِّ مرحلة التركيب النموذجي، دورا فاعلا ِِّ توضيح قيام النموذج على مجموعة من النظريات والقوانين (Giere, 2010) بالذكر أن التدريس بالنماذج يركز على تقديم الجانب

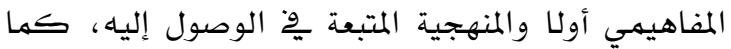
يستخدم الجانب الرياضي لمعالجة المعلومات ومساندة الفهم (Halloun, 2004)، الذي كان له دور بِّ مراعاة الفروق الفردية بين الطلبة وأنهاط تعلمهم. أها عدم وجود فروق دالة إحصائيا بين متوسطي درجات طالبات المجموعتين يّْ مجال حفظ كمية التحرك فيعزى إلى أن اكتسـاب مفاهيم حفظ كمية التحرك يحتاج إلى ربط بين عدد من المفاهيم الفيزيائية التي درسنها الطالبات مسبقا والتي اتضح أنهن يمتلكن تصورات

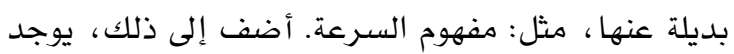
لبس لدى طالبات المجموعتين يخٌ التقريق بين الكميات العددية والكميات المتجهة؛ إذ أن السرعة كمية متجهة إلا أنها من عوامل طاقة الحركة والتي تعد كمية عددية. كما أن بعض أمثلة وتطبيقات حفظ كمية التحرك تحتاج إلى تطبيق مفاهيم حفظ الطاقة وحفظ لـ كمية التحرك معا، وهذا ما يجعل اكتساب مفاهيم حفظ كمية التحرك يْ حاجة إلى وقت أطول لمعالجة التصورات البديلة السابقة لديهن ومن ثم بناء مفاهيم كمية التحرك والدفع ومعرفة العلاقة بينهما وصولا إلى مبدأ حفظ كمية التحرك.

ولمناقشة التصورات البديلة ِِّ حفظ الطاقة وكمية التحرك لدى طالبات الصف الحادي عشر ، وللإجابة عن السؤال الثاني من الدراسة الذي ينص على "ما أثر التدريس بطريقة النمذجة بِ تعديل التصورات البديلة لدى طالبات الصف الحادي عشر عن مفاهيم حفظ الطاقة وكمية التحركى"، نستعرض أولا المفاهيم

الرئيسة والأسئلة ذات العلاقة كما يوضحها جدول بال بعد ذلك تم حساب نسب اختيار الطالبات للبديل الصحيح وِ الأسئلة وِ المجموعتين التجريبية والضابطة، قبل إجراء المعالجة وبعدها ، وكذلك الاكتساب المعياري (g) ويوضتح جدول r تلك النسب. 
جدول rاكباب

نسب البديل الصحيح القبلية والبعدية لكل سوأل، والاكتساب المعياري للمجموعتين التجريبية والضابطة

\begin{tabular}{|c|c|c|c|c|c|c|}
\hline \multicolumn{3}{|c|}{ ضابطة } & \multicolumn{3}{|c|}{ تجريبية } & \multirow{2}{*}{ رقلم السوال } \\
\hline "g" الاكتساب المعياري & بعدي (\%) & قبلي (\%) & "g" الاكتساب المعياري & بعدي (\%) & قبلي (\%) & \\
\hline$\cdot, \cdot \wedge-$ & $\varepsilon 1, r$ & $\varepsilon 0, V$ & $\cdot, \leqslant \wedge$ & $T Y, Y$ & $r V, r$ & 1 \\
\hline$\cdot, r \cdot$ & $r r, q$ & $\varepsilon, r$ & $\cdot, \leqslant 1$ & $\sum \wedge, 9$ & $1 r, 7$ & r \\
\hline$\cdot$, ro & $\varepsilon 1, r$ & $r_{1}, V$ & $\cdot, \leqslant \vee$ & 7. & ro & $r$ \\
\hline$\cdot, .0-$ & $\wedge, \vee$ & ir & . TO & $r v, \wedge$ & $\varepsilon, 0$ & $\varepsilon$ \\
\hline$\cdot, Y \wedge-$ & $r \cdot, \varepsilon$ & $\varepsilon 0, V$ & $\cdot, 0 \cdot-$ & $\varepsilon r, r$ & $71, \varepsilon$ & 0 \\
\hline$\cdot, 1 \mathrm{r}$ & $r_{1}, V$ & $1 \cdot, 9$ &..$\times 9$ & $\varepsilon r, r$ & $1 \wedge, r$ & 7 \\
\hline$\cdot, I V$ & $r 7,1$ & $1 \cdot, 9$ & $\cdot, Y \wedge$ & $r, 1$ & $\varepsilon, 0$ & v \\
\hline. .17 & rI,,$V$ & 7,0 & $\cdot, \imath \wedge$ & 01,1 & $7, \wedge$ & $\wedge$ \\
\hline$\cdot, \cdot \wedge$ & ir & 19,7 & $\cdot, .0$ & $\mid v, \Lambda$ & $1 r, 4$ & 9 \\
\hline . & YI,V & YI,,$V$ & $\cdot, I V$ & $r v, \Lambda$ & ro & 1. \\
\hline$\cdot, \cdot 9$ & $10, r$ & 7,0 & $\cdot, 1 K$ & $I V, \Lambda$ & $7, \wedge$ & 11 \\
\hline$\cdot, .0-$ & $10, r$ & 19,7 & $\cdot, \cdot r$ & $I V, \wedge$ & 10,9 & ir \\
\hline$\cdot, \cdot r$ & $r \varepsilon, \wedge$ & $r, T$ & $\cdot, \varepsilon Y-$ & $r \wedge, q$ & o. & IT \\
\hline . & $r q, 1$ & $r q, 1$ & $\cdot, I V$ & $r v, \Lambda$ & ro & $1 \varepsilon$ \\
\hline$\cdot, 0 \leqslant$ & $7 ., 9$ & $10, r$ & $\cdot, r \leqslant$ & $r v, \wedge$ & $1 \wedge, r$ & 10 \\
\hline$\cdot, \leqslant 1$ & $\varepsilon r, 0$ & $\varepsilon, r$ & $\cdot, \Gamma \cdot$ & $\varepsilon \varepsilon, \varepsilon$ & $r \cdot, 0$ & 17 \\
\hline • & $r r, q$ & $\wedge, 9$ & •, r & $r v, \wedge$ & 9,1 & IV \\
\hline$\cdot, 1$ & $r q, 1$ & Tr,T & $\cdot, .0-$ & $r v, \Lambda$ & $\varepsilon \cdot, q$ & 11 \\
\hline . & 7,0 & 7,0 & $\cdot, \cdot 7-$ & 10,7 & $r \cdot, 0$ & 19 \\
\hline , ro & $0 \leqslant, \Gamma$ & $r q, 1$ & $\cdot, \leqslant १-$ & $T r, r$ & $V \varepsilon, V$ & $r$. \\
\hline$\cdot, \cdot \varepsilon$ & $\pi$ & $\pi$ & $\cdot, \Gamma \wedge$ & 7. & O & المتوسط \\
\hline
\end{tabular}

الذي أحس بالتعب أكثر، هو من بذل شغلا أكبر، وهذا الاستعهال اليومي لمفهوم الشغل يتعارض هع المفهوم

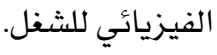
كما تشير استجابات الطالبات على السؤال 7 إلى صعوبة إدراك الطالبات بأن الشغل المبذول بواسطة الجاذبية أدى إلى تغيير اتجاه السرعة وبالتالي تغير طاقة حركتها وفق نظرية الشغل -الطاقة ، وأن ثبات مقدار السـرعة لا يعني ثبات اتجاهها ، إذ مثلت نسبة استجابة الطالبات على

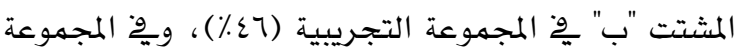

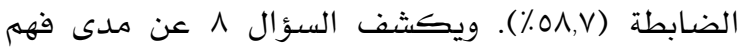
الطالبات لمفهوم الشغل باعتباره حاصل ضرب القوة والإزاحة، إذ أشارت ما نسبته (1,1\%) من استجابات الطالبات پِ المجموعة التجريبية على استيعابهن لمفهوم

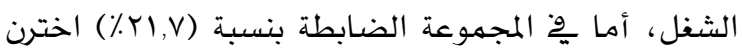
البديل الصحيح، ويعود ذلك إلى أن الطالبات يعتقدن أن
ولمعرفة مزيد من التفاصيل عن التصورات البديلة لدى الطالبات حول مفاهيم حفظ الطاقة وكمية التحرك والصعوبات التي تواجهها الطالبات عند توظيف هذه المفاهيم، يوضح الجدول وِّ ملحق ا نسب جميع البدائل وِّ المجموعتين التجريبية والضابطة قبليا وبعديا، ويعد البديل الصحيح هو البديل الذي تحته خط.

اولا: الشغل المبذول بواسطة الجاذبية : تسبر الأسئلة 1، 7، A، IV فهم الطالبات للشغل المبذول بواسطة الجاذبية، الذي يعد وظيفة جوهرية من وظائف النموذج الأول ومكونا أساسيا للتركيب الخارجي للنهوذج. إذ يركز السؤال ا على أن الشغل المبذول بواسطة الجاذبية يعتمد على طول المسار، وقد تقوقت المجموعة التجريبية على

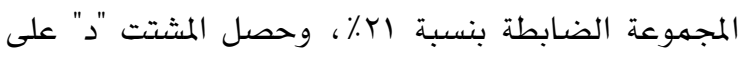
نسبة ^^,^\% المجموعة الضابطة، إذ يتبادر إلى ذهن الطلبة أن الشخص إنص 


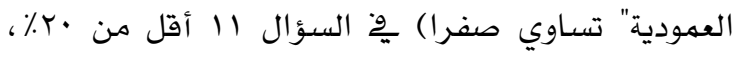
وبذلك يضيف السؤال تصورا بديلا آخر هو أن الكتلة

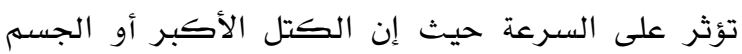
الأثقل يمتلك سرعة أكبر هِّ الأسفل لأن الوزن الأكبر

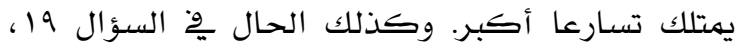
حيث أن نسب استجابات الطالبات أيضا بِّ2 المجهوعتين

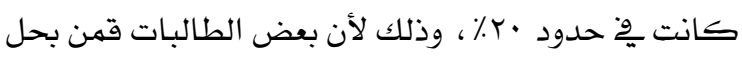
السؤال على أنه سؤال ِِّ المقذوفات، إذ أن اختيار البديل

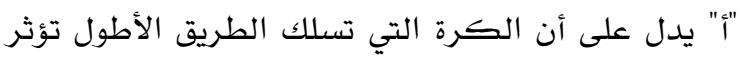

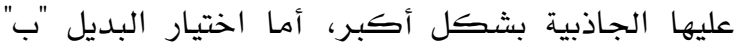

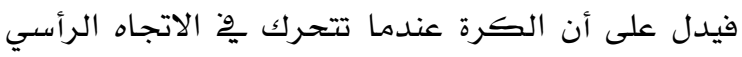
تقل سرعتها ، لأن محصلة حركتها تكون فِّون الاتجاه

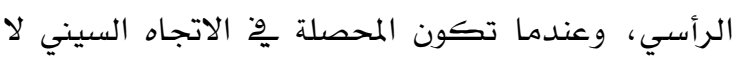

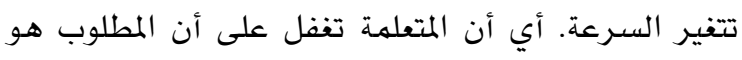

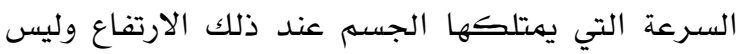

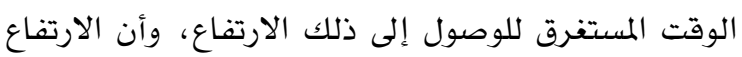

$$
\text { هو نفسها لجميع الكرات. }
$$

إن قلة نسب استجابة الطالبات للبديل الصحيح بِّ2 السؤال

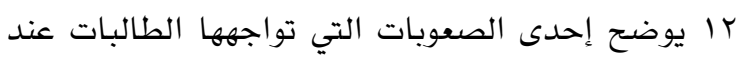
حل مسائل قائمة على مبدأ حفظ الطاقة الميكيانيكة، الماندان والمتمثلة وِّ عدم القدرة على التمييز بين مفاهيم الطاقة

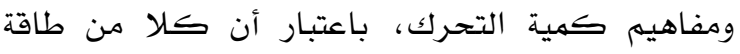

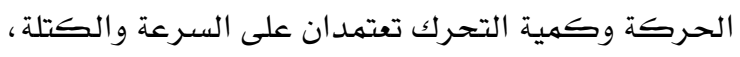

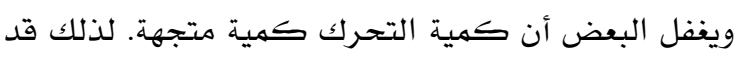

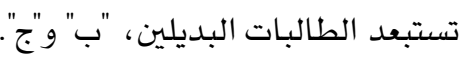
ثالثًا: مفهوم النظام: إن السؤالين ؟، و. إوضحان مدى اكتساب الطالبات لمفهوم النظام، الذي يعد من المفاهيم الأساسية التي تغفل بعض المناهج الدراسية عن توضيحها. حيث إن أعلى نسبة مشتت تم اختياره من قبل الطالبات

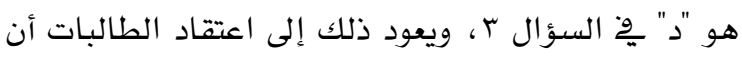
طاقة الحركة وكمية التحرك محفوظة لكل مكون

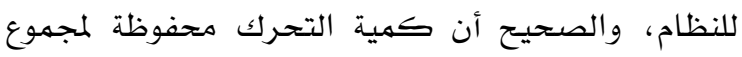

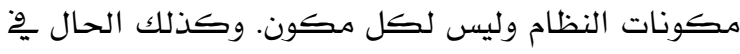

مجموع طاقات حركة الأجسام پِّ التصادمات المرنة.

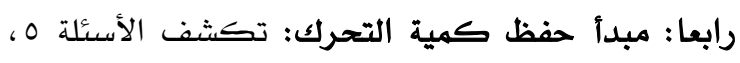

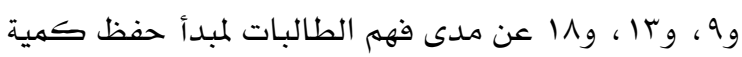

بذل قوة أكبر يعني بذل شغل أكبر، أي أنهن لا يستطعن

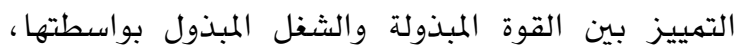

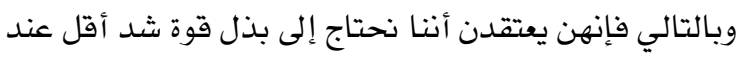

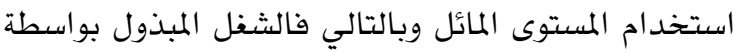

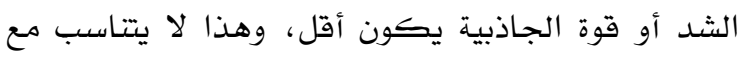

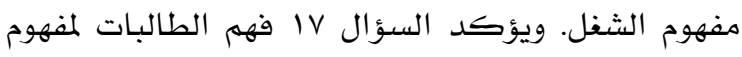

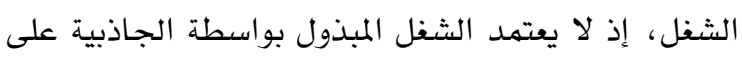

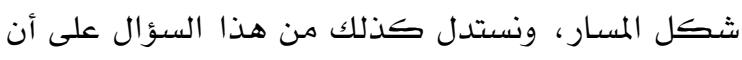
الطالبة لا تستطيع الربط بين الشغل المبذول وطاقة الحركة. كما أن استجابة طالبات المجموعة الضابطة

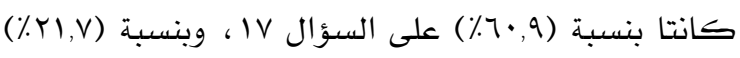

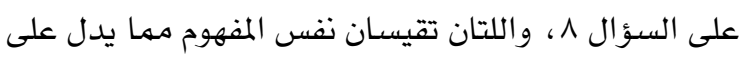
عدم وجود جسم منظم من المعرفة لديهن عن مفهوم الشغل المبذول بواسطة الجاذبية الأرضية.

ثانيا: مبدأ حفظ الطاقة الميكيانيكية: تقيس الأسئلة

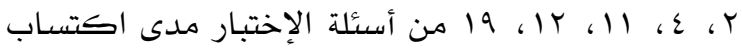

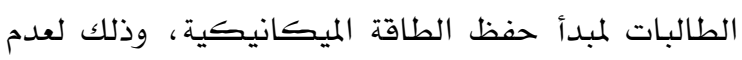
وجود شغل مبذول بواسطة القوى غير المحافظة (قوة الجاذبية والقوة العهودية). وكانت نسب الطالبات اللاني

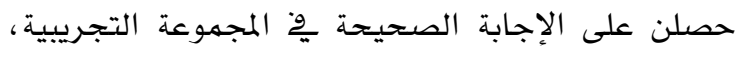

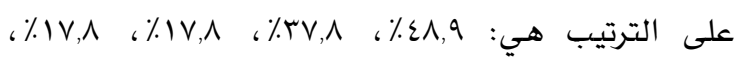

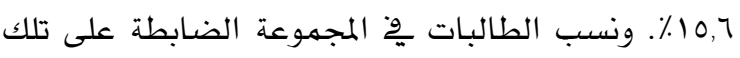

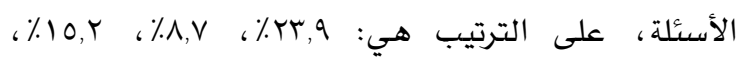
$\%, 0, \%, 10, Y$ كما أن المشتت "ب" بِّ السؤال r حصل على أعلى نسبة وِّْ المجموعتين التجريبية والضابطة فكانت على الترتيب:

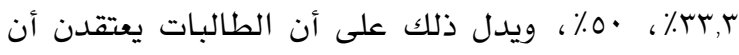

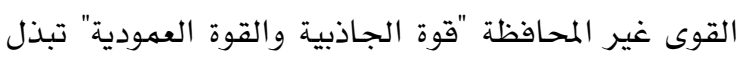
شغلا، كما أن شكل المسار يؤثر على الشغل المبذول

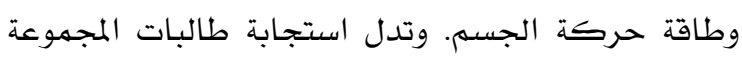

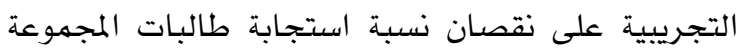

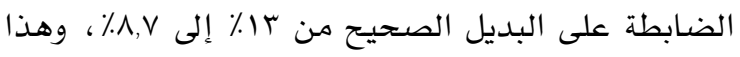

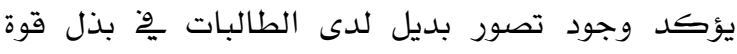

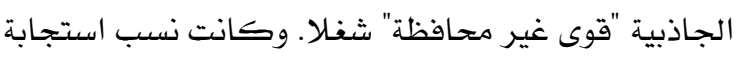
الطالبات پِّو المجموعتين على البديل الصحيح (أن الشغل

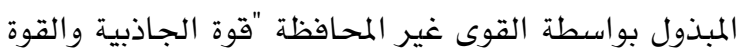


للبديل الصحيح ِِّ السؤالين هي أعلى عن •ع٪، ولكن حصل المشتت "أ" على أعلى نسبة بين المشتات بِّ السؤال 17 ، وهذا دليل على عدم فهم الطالبات بأن التفير بو

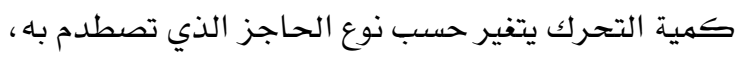

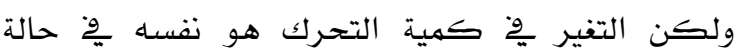
الاصطدام بجدار اسهنتي أو بكومة قش ولكن فترة

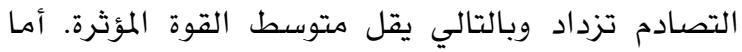
السؤال • ب فهو على مستوى الاستدلال؛ إذ يتتاول العلاقة

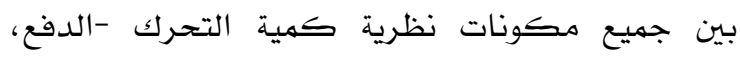

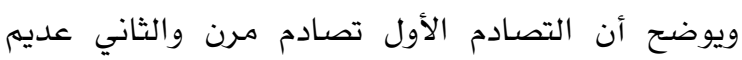

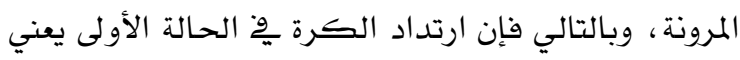
أن التغير ِِّ كمية التحرك أكبر. ونظرا لثبات الفترة الزمنية، فان القوة المؤثرة على السطح تكون أكبر أيضا.

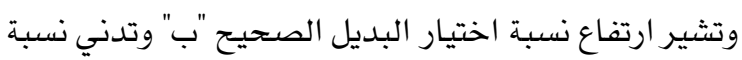

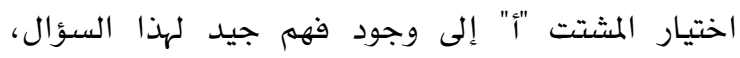

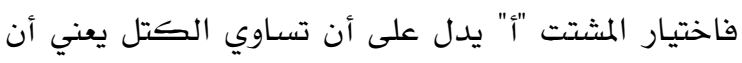

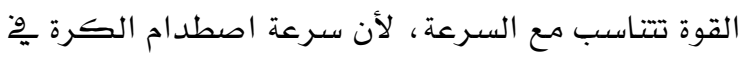
كل حالة متساو فيؤدي الى تساو متوسط القوة المؤثرة بغض النظر عن ارتدادها أو التصاقها بالسطح.

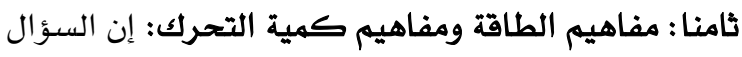
عا يوضح مدى قدرة الطالبات الربط بين مفاهيم الطاقة

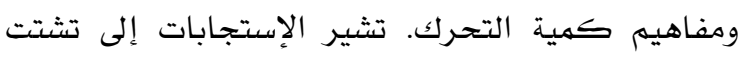

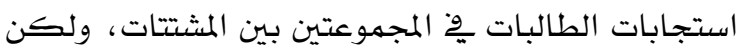
حاز البديل الصحيح على أعلى نسبة كها هو موضح بِّ2

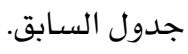

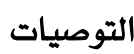

وِّْ ضوء النتائج التي توصلت إليها هذه الدراسة حول أثر التدريس بطريقة النمذجة وْ اكتساب طالبات الصف الحادي عشر للمفاهيم الفيزيائية وتصوراتهن نحوها، توصي الدراسة بها يأتي:

1. تشجيع معلهي العلوم بشكل عام والفيزياء بشكل

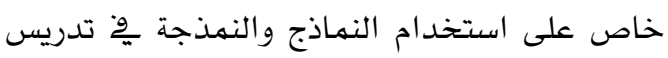
مادتهم.
التحرك. ويوضح انخفاض نسب حصول الطالبات على البديل الصحيح ِِّ السؤال 0 ِِّ المجموعتين إلى وجود

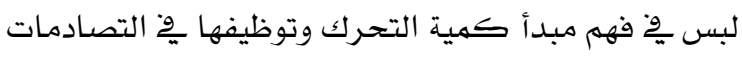
بأنواعها ، إذ تعتقد الطالبات أن كمية التحرك محفوظة

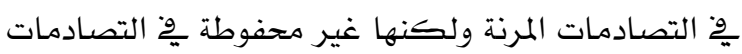
غير المرنة، كما أن البعض منهن لا يدركن أن كمية

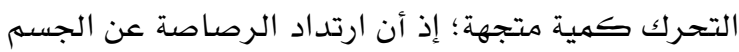

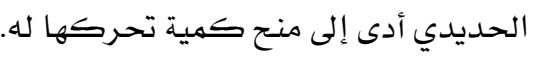

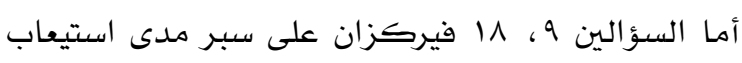

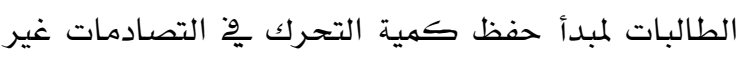
المرنة أو الانقجارات، ومن خلال استجابة الطالبات يتضح

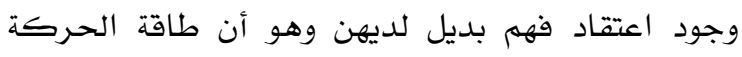

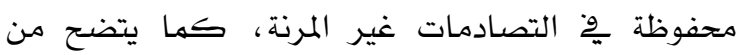

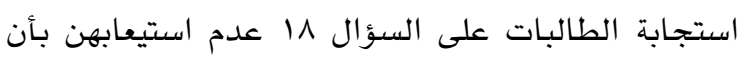
زيادة الكتلة (نتيجة تراكم قطرات المطر) يؤدي إلى الى لى

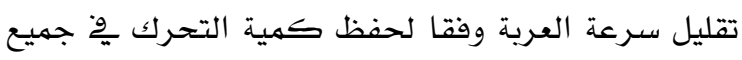

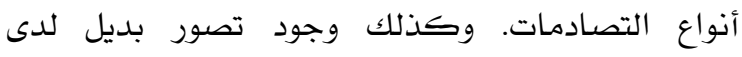
الطالبات بِّ حفظ طاقة الحركة بِّ التصادمات عديمة المرونة عند المقارنة بين اثتين من التصادمات عديمة المرونة كما بالسؤال سال خامسا : مفهوم كمية التحرك: يقيس السؤال V مدى فهم

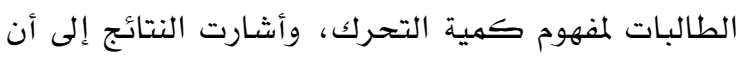

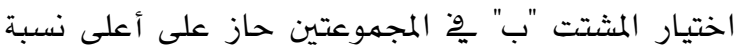
مشتت من بين المشتتات الثلاثة، ويعود ذلك إلى عدم وجود

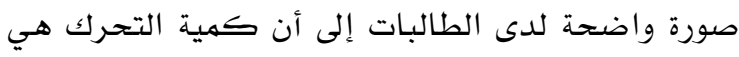

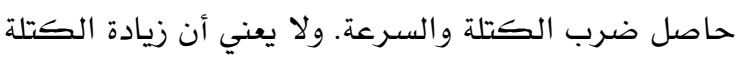

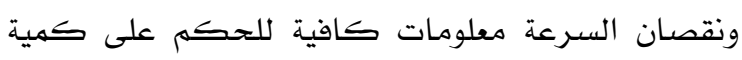

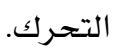

سادسا: العلاقة بين كمية التحرك والدفع: يقيس السؤال رقم 10 العلاقة بين كمية التحرك والدفع، ومبني وفق بقاتع

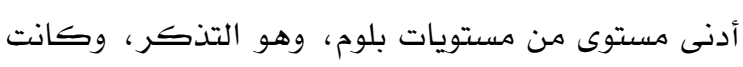

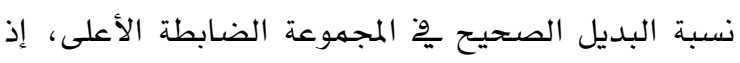

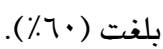
سابعا: تطبيقات نظرية كمية التحرك -الدفع: يوجد سؤالان يقيسان قدرة الطالبات على توظيف نظرية كمية التحرك -الدفع هما 17، r. إن نسبة اختيار الطالبات 
زيتون، عايث محمود (·(ب). الاتجاهات العالمية المعاصرة ِِّ مناهج العلوم وتدريسها. عمان، دار

الشـروق.

زيتون، عايث محمود (1991). أساليب تدريس العلوم،

الطبعة الأولى، عمان، دار الشروق للنشر والتوزيع

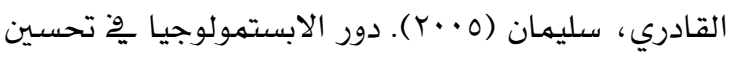

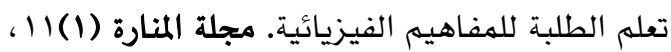

$.1 Y V-1.1$

Buckley, B. C. (2012). Encyclopedia of the Sciences of Learning.

Retrieved on 20 October, 2014 from: http://link.springer.com/referenceworkentry/10.1007 1978-1-4419-1428-6_4977\#

Chabalengula, V. M., Sanders, M. \& Mumba, F. (2012). Diagnosing students' understanding of energy and its related concepts in Biological context. International Journal of Science and Mathematics Education, 102, 241-266.

Clement, J. (1982). Students' preconceptions in introductory mechanics. American Journal of Physics, 50(1), 66-71.

Cobern, W. W. (1993). College students' conceptualizations of nature: An interpretive world view analysis. Journal of Research in Science Teaching, 30(8), 935-951.

Cutnell, J. \& Jonson, K. (2010). Introduction to physics: International student version. Hoboken, River Street: John Wiley \& Sons Pte Ltd.

Dalaklioglu, S., Demirci, N., Sekercioglue, A. (2015). Eleventh grade student's difficulties and misconceptions about Energy and Momentum concepts. International Journal on New Trends in Education and Implications, 6(1), 13-21.

George, E.A., Broadstock, M.J., Vazquez Abad, J. (2000). Learning Energy, Momentum, and Conservation Concepts withComputer Support in an Undergraduate Physics Laboratory. In B. Fishman \& S. O'ConnorDivelbiss (Eds.). Fourth International Conference of the Learning Sciences (pp. 2-3). Mahwah, NJ: Erlbaum.

Giere, R.N., (2004). How Models Are Used to Represent Reality. Philosophy of Science, 71 (5),

742-752.

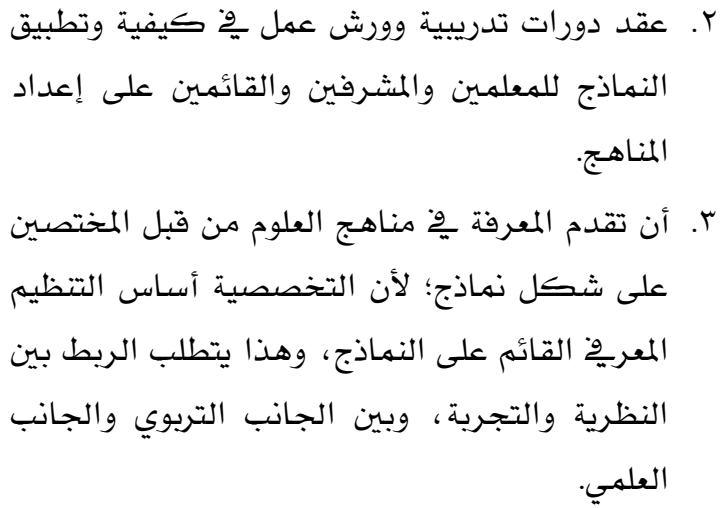

\section{References}

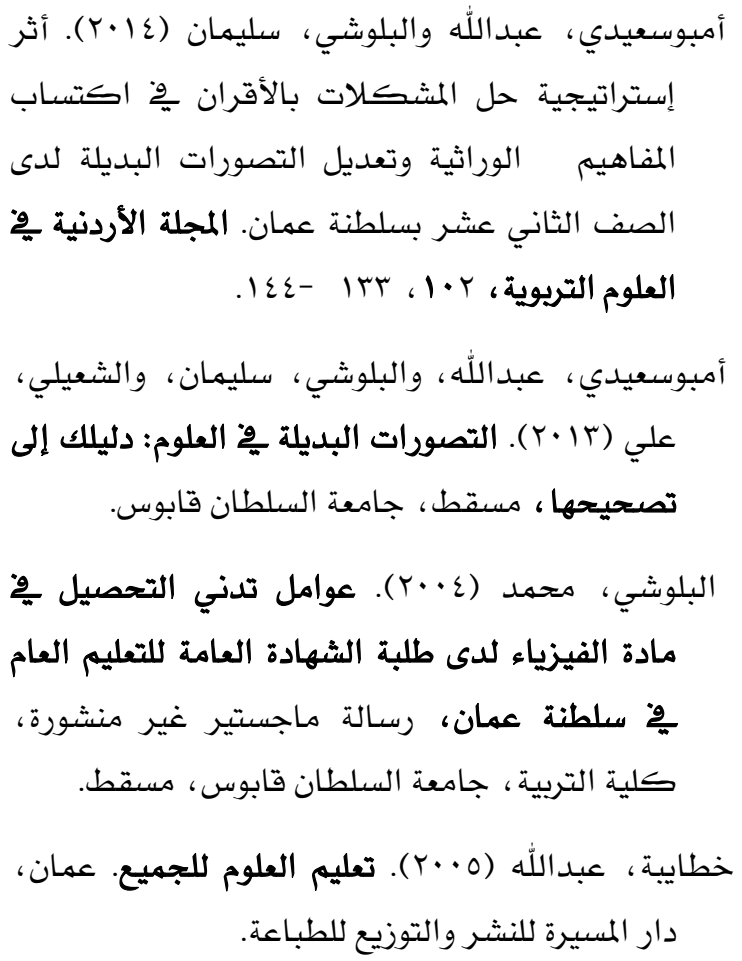


Gilbert, J., Boulter, C. \& Rutherford, M. (1998). Models in explanations, Part 1: Horses for courses? International Journal of Science Education, 20(1), 83-97.

Grahan, T. \& Berry, J. (1996). A hierarchical model of the development of student understanding of momentum. International Journal of Science Education, 18(1), 75-89.

Halloun, I.A. (1996). Schematic modeling for meaningful learning of physics. Journal of

Research in Science Teaching, 33(9), 1019-1041.

Halloun, I.A. (2004). Modeling Theoy in Science Education. New York, the Netherlands: Science \& Technology Education Library.

Halloun, I.A. (2011). Profile Shaping Education. A paradigm shift in education to empower students for success in modern life.Paper presented at $11^{\text {th }}$ IHPST Conference Proceeding, pp 337-343. Thessaloniki, Greece: IHPST.

Halloun, I.A. (2001, April). Student Views about Science: A comparative Survey. Beirut: Phoenix Series / Educational Research Center, Lebanese University.

Hammer, D. (1994). Epistemological beliefs in introductory physics. Cognition and Instruction, 122, 151-183.

Hestenes, D. (1987). Toward a modeling theory of physics instruction. Journal of American Physics, 55 5, 440-454.

Horner, M., Jeng, M. \& Lindell, R. (2007). Comparison of Teaching Methods for Energy Conservation. Paper presented at the Physics Education Research Conference, pp 161-164, Syracuse.

Jackson, J., Dukerich, L., \& Hestenes, D. (2008). Modeling instruction: an effective model for science education. Science Educator, 17(1), 10-17.

Koponen, I. (2007). Models and Modeling in Physics Education: A Critical Re-analysis of Philosophical Underpinnings and Suggestions for Revisions. Science $\mathcal{E}$ Education, 16 (7), 751-773.

Leea, C.B., Jonassenb, D., \& Teo, T (2011). The role of model building in problem solving and conceptual change. Interactive Learning Environments, 193, 247-265.

Mäntylä, T. (2011). Didactical reconstructions for organizing knowledge in physics teacher education. A cademic Dissertation, Department of physics, University of Helsinki. Finland.

Matthews, M. (1997). Introductory comments on philosophy and constructivism in science education. Science \& Education, 6(1-2), 5 - 14.

Mclldowie, E. (1995). Energy transfer. Where did we go wrong? Physics Education, 30 (4), 228230.

McDermott, L. (1997). Curriculum development and the assessment of student learning: An example from The work-energy and impulse-momentum theorems. Science Education, 2(1), 27-42.

McDermott, L. (1993). How we teach and how students learn-a mismatch? American Journal of Physics, 614, 295-298.

Mercan, F. (2012). Epistemic Beliefs about Justification Employed by Physics Studente and Faculty in Tw Different Problem Contexts. International Journal of Science Education, 34(9), 1411-1441.

National Science Teachers Association (NSTA). (1995). Scope, Sequence and Coordination of Secondary School Science. Volume 3. A High School Framework for National Science Education Standards. Washington, DC: NSTA.

New Generation of Science Standards NGSS (2013). A Framework for K-12 Science Education: Practices, Crosscutting Concepts, and Core Ideas. Retrieved on September 14, 2013 from The National Academies Press at http://www.nextgenscience.org/nextgeneration-science-standards

Ornek, F. (2008). Models in science education: applications of models in learning and teaching science. International Journal of Environmental \& Science Education, 32, 35 45.

Perkins, K.K., Adams, W.K., Pollock, S.J., Finkelstein, N. D. \& Wieman, C. E. (2004). Correlating Student Beliefs with Student Learning Using The Colorado Learning Attitudes about Science Survey. Paper 
presented at Physics Education Research Conference, 61-64. Sacramento: California.

Philippi, K.H. (2010). An examination of student understanding of the use of models in science and conceptual understanding of electricity and magnetism. Published Doctor of Philosophy, university of New Orleans, USA.

Reif, F., \& Larkin, J. H. (1991). Cognition in scientific and everyday domains: Comparison and learning implications. Journal of Research in Science Teaching, 28(9), 733-7.

Sabella, M \& Redish, E. (2007). Knowledge activation and organization in physics problem-solving. American Journal of Physics, 75(11), 1017-1029.

Sin, C., (2014). Epistemology, Sociology, and Learning and Teaching in Physics. Science Education, 98(1), 342-365.

Singh, C. \& Rosengrant. D. (2003). Multiplechoice test of energy and momentum concepts. American Association of Physics Teachers, 71(6), 607-617.

Taser, M.F, (2010, May). What part of the concept of acceleration is difficult to understand: the mathematics, the physics, or both? ZDM Mathematics Education, 42, 469-482.

Wells, M., Hestenes, D., \& Swackhamer, G. (1995). A modeling method for high school physics instruction. . American Journal of Physics, 63(7), 606-619. 
ملحق ا : النسب المئوية لجميع البائل لجميع الأسئلة في اختبار حفظ الطاقة وكمية التحرك للمجموعتين التجريبية والضابطة

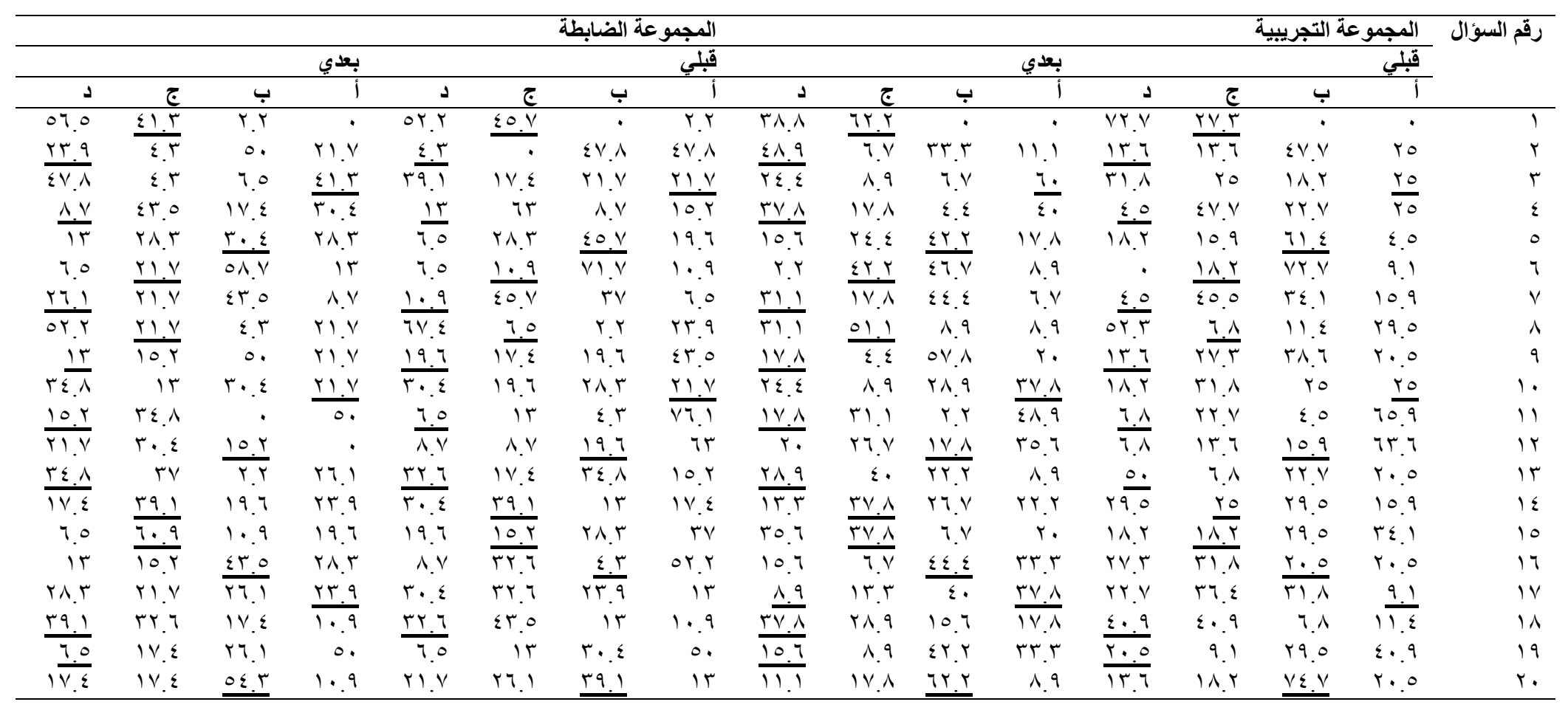

